\title{
edoc
}

Institutional Repository of the University of Basel

University Library

Schoenbeinstrasse 18-20

CH-4056 Basel, Switzerland

http://edoc.unibas.ch/

Year: 2013

\section{New insights in the regulation of skeletal muscle PGC-1 $\alpha$ by exercise and metabolic diseases}

\author{
Pérez-Schindler, Joaquín and Handschin, Christoph
}

Posted at edoc, University of Basel

Official URL: http://edoc.unibas.ch/dok/A6174378

Originally published as:

Pérez-Schindler, Joaquín and Handschin, Christoph. (2013) New insights in the regulation of skeletal muscle PGC-1 $\alpha$ by exercise and metabolic diseases. Drug discovery today. Disease models, Vol. 10, H. 2 , e79-e85. 
New insights in the regulation of skeletal muscle PGC-1 - by exercise and metabolic diseases

\section{Joaquín Pérez-Schindler and Christoph Handschin*}

Biozentrum, University of Basel, Klingelbergstrasse 50/70, 4056 Basel, Switzerland

Published in Drug Discov Today Disease Models (2013) 10(2): e79-85. DOI:

10.1016/j.ddmod.2012.12.002

Copyright (C) Elsevier; Drug Discovery Today: Disease Models 
New insights in the regulation of skeletal muscle PGC-1 $\alpha$ by exercise and metabolic diseases

Joaquín Pérez-Schindler and Christoph Handschin*

Biozentrum, University of Basel, Klingelbergstrasse 50/70, 4056 Basel, Switzerland

*Corresponding author: C. Handschin (christoph.handschin@unibas.ch) 


\section{Abstract}

Skeletal muscle energy metabolism is severely impaired in insulin resistant and type 2 diabetic patients. In particular, deregulated transcription of oxidative metabolism genes has been linked to the development of non-communicable metabolic diseases. The peroxisome proliferator-activated receptor $\gamma$ (PPAR $\gamma$ ) coactivator-1 $\alpha$ (PGC-1 $\alpha)$ is a key molecule in the regulation of oxidative metabolism in different tissues, including skeletal muscle. In this tissue, physical exercise is one of the most dominant physiological stimuli to induce PGC-1 $\alpha$. In addition, exercise training efficiently prevents the development of metabolic diseases. Hence, better knowledge about the regulation of PGC-1 $\alpha$ by exercise would significantly help to design effective treatments for these diseases.

\section{Introduction}

Physical inactivity is nowadays considered as a pandemic, representing an important etiological factor in the development of non-communicable diseases and a major cause of premature death worldwide [1,2]. The detrimental effects of a sedentary life style are largely associated with the impairment of skeletal muscle metabolic fitness, which is mainly characterized by a lower oxidative metabolism [3,4]. Even though causality of mitochondrial dysfunction in the development of insulin resistance remains controversial, insulin-resistant and type 2 diabetic patients often present an impairment of skeletal muscle oxidative metabolism [5]. Is important to note that a pathological decrease in skeletal muscle insulin sensitivity is sufficient to promote the development of the metabolic syndrome [6,7]. Therefore, the metabolic remodeling of skeletal muscle cells plays a central role in the pathogenesis of metabolic diseases like type 2 diabetes.

The development of obesity and type 2 diabetes largely depends on different environmental factors, including the balance between physical activity and inactivity [8-10]. At the transcriptional level, the peroxisome proliferator-activated receptor $\gamma$ (PPAR $\gamma$ ) coactivator-1 $\alpha$ (PGC-1 $\alpha$ ) plays a key role in exercise-linked mitochondrial remodeling $[11,12]$. This coactivator regulates a specific transcriptional network, in which the estrogen related receptors (ERRs), nuclear respiratory factors (NRFs) and PPAR $\delta$ are the principal transcription factors involved in the enhancement of oxidative metabolism [11]. Consistent with its metabolic function, impairment of PGC-1 $\alpha$ expression and activity have been identified as potential pathogenic factors in the development of insulin resistance and type 2 diabetes $[5,13,14]$. The expression of PGC-1 $\alpha$ is sensitive to muscle contraction with high levels in trained subjects and a strong down-regulation in muscle from people with spinal cord injury [15]. Here, we review the specific mechanism by which different types of physical exercise regulate skeletal muscle PGC-1 $\alpha$ and how this process is affected by non-communicable metabolic diseases.

\section{Regulation of PGC-1 $\alpha$ expression and activity by skeletal muscle contraction}

Muscle contraction modulates different signal pathways that trigger the activation or repression of a very specific subset of genes, such as the slow-oxidative or fast-glycolytic gene program observed in type 1 and 2 skeletal muscle fibers, respectively [16]. PGC-1 $\alpha$ specifically controls the expression of the slow-oxidative gene program, while the expression and activity of this coactivator is regulated by several protein kinases/phosphatases, transcription factors and coregulators in response to muscle contraction [12].

\section{Transcriptional regulation of PGC-1 $\alpha$ in skeletal muscle}


The human and mouse PGC-1 $\alpha$ promoters contain a number of binding sites for different transcription factors, including PPARs, myocyte enhancer factor 2 (MEF2), activating transcription factor 2 (ATF2), forkhead box 01 (FOXO1), p53 and others [17-19]. Primarily however, muscle contraction mediates the activation of this promoter via the MEF2 binding sites and the CAMP response element (CRE) [20]. The transcriptional activity of MEF2 can be increased by PGC-1 $\alpha$ or repressed by the histone deacetylase 5 (HDAC5) [18,21]. Muscle contraction can decrease the inhibitory effect of HDAC5 through its phosphorylation by protein kinase D (PKD), 5'AMP-activated protein kinase (AMPK) and $\mathrm{Ca}^{2+} /$ calmodulin-dependent protein kinase II (CaMKII) [22-24], representing an essential process in the activation of the PGC-1 $\alpha$ promoter [22]. In contrast, PGC-1 $\alpha$ is strongly induced by exercise (see below) and its positive effect on MEF2 has been suggested to activate a positive feedback loop, thus increasing its own transcription [18]. CRE binding protein (CREB) and ATF2 are the main transcription factors recruited to the CRE site in the PGC-1 $\alpha$ promoter in response to muscle contraction [22]. Interestingly, both MEF2 and ATF2 are activated by p38 mitogen-activated protein kinase (MAPK) $[25,26]$, indicating that this protein kinase is intimately involved in the regulation of PGC-1 $\alpha$ transcription. Consistently, Pogozelski et al. [27] demonstrated that muscle-specific deletion of p38 $\gamma$ MAPK completely blocked the up-regulation of PGC-1 $\alpha$ induced by exercise. Accordingly, activation of p38 MAPK by reactive oxygen species (ROS) in response to exercise training is indeed an important mechanism regulating PGC-1 $\alpha$ expression in skeletal muscle $[28,29]$. Similarly, PKD activation is required for the activation of the PGC-1 $\alpha$ promoter since the expression of a catalytic inactive form of PKD can fully prevent the effects of muscle contraction on PGC-1 $\alpha$ expression [22]. In contrast to P38 MAPK and PKD, the relevance of AMPK and CaMKII in the regulation of PGC-1 $\alpha$ expression by exercise has been more difficult to elucidate. For instance, muscle-specific deletion of the $\beta 1$ and $\beta 2$ subunits of AMPK significantly decreases the expression and activity of different mitochondrial enzymes during basal conditions, though it paradoxically results in higher levels of PGC-1 $\alpha$ mRNA [30] while, to our knowledge, no studies exist that investigated the requirement for CaMKII for exercise-mediated regulation of PGC-1 $\alpha$ expression. Interestingly, endurance exercise induces the activation p53 [31], suggesting that it could promote the activation of the PGC-1 $\alpha$ promoter. Moreover, a single bout of low-intensity endurance exercise results in higher mRNA levels of the transcription factors PPAR $\delta$ and FOXO1 [32], both of which have been described to bind to the PGC- $1 \alpha$ promoter $[17,19]$. In particular, PPAR $\delta$ in complex with RXR $\alpha$ can bind the PPAR response elements (PPRE) of the PGC-1 $\alpha$ promoter and increase its transcriptional activity [19]. Considering that PGC-1 $\alpha$ co-activates PPAR $\delta$ [33], this could represent another positive feedback loop in the transcriptional regulation of this coactivator, but is currently unknown whether PPARS plays a significant role during endurance exercise.

More recently, novel mechanisms involving epigenetic modifications of the DNA and posttranscriptional regulation by microRNAs have been proposed to modulate PGC- $1 \alpha$ transcription in response to exercise and pathological conditions. Interestingly, Barrès et al. [34] demonstrated that the DNA methyltransferase 3B (DNMT3B) could repress PGC-1 $\alpha$ expression through non-CpG dinucleotide methylation of its promoter in human skeletal muscle. Importantly, the PGC-1 $\alpha$ promoter was found to be hyper-methylated in skeletal muscle from impaired glucose-tolerant and type 2 diabetic patients, showing a negative correlation with PGC-1 $\alpha$ mRNA levels and a decreased expression of several mitochondrial enzymes [34]. Inversely, acute exercise in human skeletal muscle and ex-vivo electrical stimulation of mouse skeletal muscle induce a significant decrease in the methylation levels of the PGC-1 $\alpha$ promoter, which precede the increase in its transcript levels [35]. 
Finally, physical activity and inactivity induce the down- and up-regulation of the microRNA miR-696, respectively, which negatively regulates PGC-1 $\alpha$ expression in skeletal muscle [36]. Thus, overall, the PGC-1 $\alpha$ promoter contains a high number of different transcription factor binding sites that control PGC-1 $\alpha$ expression while the activation of additional signal pathways represent a potential mechanism by which PGC-1 $\alpha$ transcript levels are further fine-tuned (Fig. 1A).

\section{Post-translational regulation of PGC-1 $\alpha$ in skeletal muscle}

In skeletal muscle, PGC-1 $\alpha$ is a direct target of both AMPK and p38 MAPK, which both phosphorylate it at distinct sites and hereby induce its activation $[37,38]$. Interestingly, it has been suggested that p38 MAPK-mediated phosphorylation and thereby stabilization of the PGC-1 $\alpha$ protein precede transcriptional induction of PGC-1 $\alpha$ gene expression in contracting muscle [39]. The balance between the acetylation and deacetylation of PGC-1 $\alpha$ is controlled by the deacetylase sirtuin 1 (SIRT1) and the acetyl transferase general control of amino-acid synthesis 5 (GCN5), respectively [40,41]. SIRT1mediated deacetylation activates PGC-1 $\alpha$ whereas GCN5 activity reverses this effect $[40,41]$. Interestingly, activation of AMPK is a prerequisite for SIRT1-controlled deacetylation of PGC-1 $\alpha$ in response to exercise [42]. Recently however, the relevance of SIRT1 for the modulation of muscle PGC-1 $\alpha$ acetylation by exercise has been questioned since the skeletal muscle-specific genetic inactivation of SIRT1 did not affect deacetylation of PGC-1 $\alpha$ and subsequent induction of its target genes in mouse skeletal muscle after acute and chronic exercise [43]. In this study, reduced interaction with GCN5 has been proposed as the main mechanism controlling the acetylation status

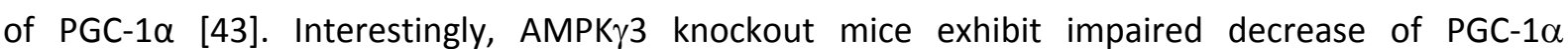
acetylation levels induced by exercise [42], suggesting a potential role of AMPK in the regulation of PGC- $1 \alpha$ acetylation by GCN5 rather than its deacetylation by SIRT1.

\section{Effects of low- vs. high-intensity exercise on PGC-1 $\alpha$}

\section{Endurance exercise}

Physical activity represents one of the strongest physiological stimuli to promote skeletal muscle oxidative metabolism and stimulate PGC-1 $\alpha$ expression. Initially, studies performed in rat skeletal muscle showed that 3 to 7 days of low-intensity swimming training could efficiently induce a $\sim 2$ fold increase in PGC-1 $\alpha$ mRNA and protein content $[44,45]$. Acutely, the completion of a total of 6 hours of low-intensity swimming or running resulted in a significant 2 to 7 fold increase in PGC-1 $\alpha$ transcript and protein levels [45-47]. Similar to rodents, a single bout of endurance exercise also results in a 4 to 12 fold increase of PGC-1 $\alpha$ mRNA in human skeletal muscle, an effect that is observed immediately after exercise up to 3 to 8 hours. Later, PGC-1 $\alpha$ expression returns to basal levels within 4 to 24 hours post-exercise [31,32,48-51]. The magnitude by which acute endurance exercise modulate human skeletal muscle PGC-1 $\alpha$ protein content is not well known, with some studies showing no increase 3 hours post-exercise [31,49] and others reporting a slight increase after endurance exercise to exhaustion [51], indicating that the intensity at which exercise is performed could be an important factor. However, in humans, endurance training over a period of 6 weeks induces a significant $\sim 2$ fold increase of both mRNA and protein levels of muscle PGC-1 $\alpha$ [52]. Interestingly, the activation of an alternative promoter of PGC-1 $\alpha$ leads to the expression of different splice variant of this coactivator (PGC-1 $\alpha-a$, PGC-1 $\alpha-b$ and PGC-1 $\alpha-c$ ) in skeletal muscle $[53,54]$. Of these three transcript variants, PGC-1 $\alpha-b$ exhibits a higher sensitivity to exercise and was indeed strongly up-regulated by endurance exercise in mouse skeletal muscle [53,54]. 
Furthermore, there is some evidence implying cytoplasmic-nuclear translocation to contribute to the regulation of PGC-1 $\alpha$, however this concept remains controversial. Interestingly, a splice variant called novel truncated PGC-1 $\alpha$ (NT-PGC-1 $\alpha$ ), which lacks key domains in nuclear localization, is thought to shuttle between cytoplasm and the nucleus [55]. Other studies imply that in rat skeletal muscle, low-intensity swimming significantly increased the nuclear abundance of "normal" PGC-1 $\alpha$ immediately after exercise [39]. In human skeletal muscle, a similar effect was reported, in which a $\sim 54 \%$ increase in nuclear PGC-1 $\alpha$ has been detected immediately after a single bout of endurance exercise [56]. In these studies, PGC-1 $\alpha$ nuclear translocation after endurance exercise correlates with an increase in the phosphorylation levels p38 MAPK and its substrate ATF2 [39,56]. Interestingly, whereas McGee \& Hargreaves [57] did not detect PGC-1 $\alpha$ nuclear translocation after a single bout of endurance exercise, they found a decrease and increase in the interaction between HDAC5 and PGC$1 \alpha$ with MEF2, respectively. Moreover, the exchange between HDAC5 and PGC-1 $\alpha$ was also associated with an increase in p38 MAPK and MEF2 phosphorylation [57]. Furthermore, endurance exercise has been recently described to also increase PGC-1 $\alpha$ levels in the mitochondrial matrix immediately after and 3 hours post-exercise [58]. Specifically, PGC-1 $\alpha$ was detected in complex with the mitochondrial transcription factor A (TFAM) at the D-loop region of the mitochondrial DNA in the mitochondrial matrix and this interaction was further enhanced by endurance exercise [58]. Therefore, endurance exercise activates a broad spectrum of proteins involved in the expression, activation and maybe also the cellular localization of PGC-1 $\alpha$.

\section{Low-volume high-intensity interval training}

During the last years, low-volume, high-intensity interval training (HIT) has been proposed as a timeefficient alternative to traditional endurance training to improve whole body and skeletal muscle oxidative metabolism [59]. For example, 6 weeks of low-volume HIT result in a higher $\mathrm{VO}_{2 \text { peak, }}$ lower respiratory exchange ratio during exercise and increased activity of different mitochondrial enzymes (e.g. citrate synthase) in skeletal muscle [60]. Interestingly, the coactivator PGC-1 $\alpha$ has also been considered as a potential mediator of the adaptations of energy metabolism to this kind of exercise training [59]. Accordingly, similar to low-intensity swimming, a single bout of high-intensity swimming in rats also induces a robust increase in skeletal muscle PGC-1 $\alpha$ protein levels [61]. Importantly, in humans, a single bout of low-volume HIT has been showed to promote a 2 to 10 fold increase in skeletal muscle PGC-1 $\alpha$ mRNA 3 to 4 hours post-exercise [31,62-64]. It seems that the transient increase in PGC-1 $\alpha$ mRNA after HIT precedes the subsequent increase of its protein levels 24 hours post-exercise [63,64]. In fact, 3 hours after acute HIT, no increase in PGC-1 $\alpha$ protein is detected $[31,62,64]$, further suggesting that mRNA levels need to be elevated before an increase at the protein level can be observed. In the long term, cycling low-volume HIT over a period of 2 to 6 weeks can induce a significant $\sim 2$ fold increase in total and nuclear muscle PGC-1 $\alpha$ protein levels in recreationally active and sedentary subjects, thus to a similar extent as classical endurance training $[60,65,66]$. Even though HIT and endurance exercise result in similar effects on PGC-1 $\alpha$ expression [60], the fact that the training volume during HIT is substantially lower strongly suggests that PGC-1 $\alpha$ is highly sensitive to exercise intensity. Recent published data from rodents and humans studies further support this idea showing that PGC-1 $\alpha$ mRNA is increased to a higher extent by high-intensity compared to low-intensity exercise $[53,67,68]$.

Both chronic and acute low-volume HIT have been claimed to stimulate the translocation of PGC-1 $\alpha$ from the cytosol to the nucleus, but different to endurance exercise, the acute effect is only detected 
3 hours post-exercise in HIT $[64,66]$. The translocation of PGC-1 $\alpha$ induced by HIT is associated with higher p38 MAPK phosphorylation levels [64]. Interestingly, besides p38 MAPK, acute HIT also increases AMPK, CaMKII and p53 phosphorylation in skeletal muscle $[31,62,64,69]$. Therefore, acute and chronic HIT activate the same signal pathways as endurance exercise (Fig. 1A), but it has been proposed that exercise at high intensity results in stronger effects overall. In fact, Egan et al. [68] compared cycling exercise trials at 40 and $80 \%$ of the $\mathrm{VO}_{2 \text { peak }}$ and showed that only high-intensity exercise induced a significant increase in AMPK, CaMKII, ATF2 and HDAC5 phosphorylation in skeletal muscle. Moreover, though both low- and high-intensity cycling increased PGC-1 $\alpha$ mRNA, the effects of cycling at $80 \%$ of the $\mathrm{VO}_{2 \text { peak }}$ were significantly higher than that at $40 \%$ of the $\mathrm{VO}_{2 \text { peak }}$ [68]. Interestingly, HIT elevates SIRT1 expression in skeletal muscle [66], suggesting that this kind of training modulates PGC- $1 \alpha$ acetylation. Also similar to endurance training, HIT upregulates PPAR $\delta$ in skeletal muscle [63]. Finally, high-intensity exercise is more efficient in decreasing the methylation levels of the PGC-1 $\alpha$ promoter [35]. These data suggest that the activation of additional signal pathways in response to high-intensity compared to low-intensity exercise would promote a higher increase of PGC-1 $\alpha$ activity and expression, representing thus a more efficient strategy in terms of time and potency compared to traditional endurance training.

\section{Impairment of exercise effects on PGC-1 $\alpha$ in metabolic diseases}

Low expression levels of skeletal muscle PGC-1 $\alpha$ are characteristic of physically inactive people and type 2 diabetes patients $[3,14,15,70]$. Accordingly, modulation of the PGC-1 $\alpha$ axis in skeletal muscle in metabolic diseases has been proposed to be impaired at different levels. De Filippis et al. [71] demonstrated that insulin resistant skeletal muscle is also "exercise resistant", since insulin resistant people exhibit diminished and delayed increase of PGC-1 $\alpha$ expression in skeletal muscle after acute high-intensity exercise. However, endurance exercise at $40 \%$ or $70 \%$ of the $\mathrm{VO}_{2 \max }$ results in a normal increase in PGC-1 $\alpha$ mRNA in human skeletal muscle from obese and type 2 diabetes patients [72]. Interestingly, only 7 days of bed rest completely blunt the induction of PGC-1 $\alpha$ by acute exercise in human skeletal muscle [73]. In animals, an attenuated or abolished up-regulation of PGC-1 $\alpha$ mRNA and its target genes after chronic exercise has been reported in skeletal muscle from both mouse and rat models suffering from the metabolic syndrome $[74,75]$. The mechanism behind this response has been linked to impaired activation of AMPK by exercise, for example in acutely exercised mice fed a high fat diet [76]. In humans however, diminished induction of PGC-1 $\alpha$ by high-intensity exercise was associated with normal activation of AMPK in insulin resistant subjects [71], while normal induction of PGC- $1 \alpha$ by low-intensity exercise was associated with impaired activation of AMPK in obese and type 2 diabetes patients [72]. Interestingly though, skeletal muscle from ob/ob mice shows no increase in AMPK phosphorylation, SIRT1 protein and PGC-1 $\alpha$ mRNA levels after chronic exercise [75]. Furthermore, the decrease of PGC-1 $\alpha$ acetylation induced by endurance training was impaired in $o b / o b$ mice skeletal muscle [75]. In $o b / o b$ mice skeletal muscle, ex vivo muscle contraction induces a slightly attenuated increase of p38 MAPK phosphorylation even though the effect was not significant compared to that observed in wild type animals [77]. However, despite of the lower levels of PGC-1 $\alpha$ expression in type 2 diabetes patients [14], p38 MAPK phosphorylation seems to be elevated [78]. Altogether it appears that the effects of physical exercise on PGC-1 $\alpha$ are negatively affected by metabolic diseases, affecting the expression and activation of this coactivator (Fig. 1A).

\section{Conclusions}


Low- and high-intensity exercise positively modulates PGC-1 $\alpha$ activity and expression through different signal pathways, in which p38 MAPK seems to play a central role (Fig. 1A). This response to exercise seems to be impaired by physical inactivity and metabolic diseases (Fig. 1A). Upon activation, PGC-1 $\alpha$ not only enhance oxidative metabolism, but it also prevent muscle atrophy and improves ROS detoxification among other effects (Fig. 1B). Hence, the modulation of this coactivator in muscle cells is an attractive approach for the treatment of metabolic diseases [79], but it should be noted that as part of exercise-induced muscle plasticity, PGC-1 $\alpha$ also enhances anabolic process such as de novo lipogenesis [80] (Fig. 1B). Accordingly, elevation of muscle PGC-1 $\alpha$ in sedentary, high fatfed mice accelerates the development of insulin resistance [81]. Importantly however, when combined with bona fide exercise, PGC-1 $\alpha$ over-expression seems to be an efficient strategy to improve metabolic fitness under pathological conditions [82,83]. Importantly, HIT appears to be a more efficient approach to induce PGC-1 $\alpha$ in skeletal muscle and improve whole body energy metabolism [59]. In fact, low-volume HIT efficiently reduces blood glucose levels in type 2 diabetic patients [84], further supporting its therapeutic effect. Therefore, the design of new therapies aiming to potentiate the effects of exercise on PGC-1 $\alpha$ represents a potential path to combat noncommunicable metabolic diseases.

\section{References}

1 Booth, F.W. et al. (2011) Lifetime sedentary living accelerates some aspects of secondary aging. Journal of applied physiology 111 (5), 1497-1504

2 Lee, I.M. et al. (2012) Effect of physical inactivity on major non-communicable diseases worldwide: an analysis of burden of disease and life expectancy. Lancet 380 (9838), 219-229

3 Bergouignan, A. et al. (2011) Physical inactivity as the culprit of metabolic inflexibility: evidence from bed-rest studies. Journal of applied physiology 111 (4), 1201-1210

4 Handschin, C. and Spiegelman, B.M. (2008) The role of exercise and PGC1alpha in inflammation and chronic disease. Nature 454 (7203), 463-469

5 Hoeks, J. and Schrauwen, P. (2012) Muscle mitochondria and insulin resistance: a human perspective. Trends in endocrinology and metabolism: TEM 23 (9), 444-450

6 Petersen, K.F. et al. (2007) The role of skeletal muscle insulin resistance in the pathogenesis of the metabolic syndrome. Proceedings of the National Academy of Sciences of the United States of America 104 (31), 12587-12594

$7 \quad$ Kim, J.K. et al. (2000) Redistribution of substrates to adipose tissue promotes obesity in mice with selective insulin resistance in muscle. The Journal of clinical investigation 105 (12), 17911797

8 Ling, C. and Groop, L. (2009) Epigenetics: a molecular link between environmental factors and type 2 diabetes. Diabetes 58 (12), 2718-2725

9 Baar, K. (2010) Epigenetic control of skeletal muscle fibre type. Acta physiologica 199 (4), 477-487

10 Lavebratt, C. et al. (2012) Epigenetic regulation in obesity. International journal of obesity 36 (6), 757-765

11 Scarpulla, R.C. et al. (2012) Transcriptional integration of mitochondrial biogenesis. Trends in endocrinology and metabolism: TEM 23 (9), 459-466

12 Lira, V.A. et al. (2010) PGC-1alpha regulation by exercise training and its influences on muscle function and insulin sensitivity. American journal of physiology. Endocrinology and metabolism 299 (2), E145-161

13 Morris, A.P. et al. (2012) Large-scale association analysis provides insights into the genetic architecture and pathophysiology of type 2 diabetes. Nature genetics 44 (9), 981-990 
14 Mootha, V.K. et al. (2003) PGC-1alpha-responsive genes involved in oxidative phosphorylation are coordinately downregulated in human diabetes. Nature genetics 34 (3), 267-273

15 Kramer, D.K. et al. (2006) Human skeletal muscle fibre type variations correlate with PPAR alpha, PPAR delta and PGC-1 alpha mRNA. Acta physiologica 188 (3-4), 207-216

16 Schiaffino, S. and Reggiani, C. (2011) Fiber types in mammalian skeletal muscles. Physiological reviews 91 (4), 1447-1531

17 Irrcher, I. et al. (2008) AMP-activated protein kinase-regulated activation of the PGC-1alpha promoter in skeletal muscle cells. PloS one 3 (10), e3614

18 Handschin, C. et al. (2003) An autoregulatory loop controls peroxisome proliferator-activated receptor gamma coactivator 1alpha expression in muscle. Proceedings of the National Academy of Sciences of the United States of America 100 (12), 7111-7116

19 Schuler, M. et al. (2006) PGC1alpha expression is controlled in skeletal muscles by PPARbeta, whose ablation results in fiber-type switching, obesity, and type 2 diabetes. Cell metabolism 4 (5), 407-414

20 Akimoto, T. et al. (2004) Real-time imaging of peroxisome proliferator-activated receptorgamma coactivator-1alpha promoter activity in skeletal muscles of living mice. American journal of physiology. Cell physiology 287 (3), C790-796

21 Lemercier, C. et al. (2000) mHDA1/HDAC5 histone deacetylase interacts with and represses MEF2A transcriptional activity. The Journal of biological chemistry 275 (20), 15594-15599

22 Akimoto, T. et al. (2008) Functional interaction of regulatory factors with the Pgc-1alpha promoter in response to exercise by in vivo imaging. American journal of physiology. Cell physiology 295 (1), C288-292

23 McGee, S.L. et al. (2008) AMP-activated protein kinase regulates GLUT4 transcription by phosphorylating histone deacetylase 5. Diabetes 57 (4), 860-867

24 McGee, S.L. et al. (2009) Exercise-induced histone modifications in human skeletal muscle. The Journal of physiology 587 (Pt 24), 5951-5958

25 Zhao, M. et al. (1999) Regulation of the MEF2 family of transcription factors by p38. Molecular and cellular biology 19 (1), 21-30

26 Cao, W. et al. (2004) p38 mitogen-activated protein kinase is the central regulator of cyclic AMP-dependent transcription of the brown fat uncoupling protein 1 gene. Molecular and cellular biology 24 (7), 3057-3067

27 Pogozelski, A.R. et al. (2009) p38gamma mitogen-activated protein kinase is a key regulator in skeletal muscle metabolic adaptation in mice. PloS one 4 (11), e7934

28 Ristow, M. et al. (2009) Antioxidants prevent health-promoting effects of physical exercise in humans. Proceedings of the National Academy of Sciences of the United States of America 106 (21), 8665-8670

29 Meier, P. et al. (2012) The impact of antioxidant supplements and endurance exercise on genes of the carbohydrate and lipid metabolism in skeletal muscle of mice. Cell biochemistry and function

30 O'Neill, H.M. et al. (2011) AMP-activated protein kinase (AMPK) beta1beta2 muscle null mice reveal an essential role for AMPK in maintaining mitochondrial content and glucose uptake during exercise. Proceedings of the National Academy of Sciences of the United States of America 108 (38), 16092-16097

31 Bartlett, J.D. et al. (2012) Matched work high-intensity interval and continuous running induce similar increases in PGC-1alpha mRNA, AMPK, p38, and p53 phosphorylation in human skeletal muscle. Journal of applied physiology 112 (7), 1135-1143

32 Russell, A.P. et al. (2005) Regulation of metabolic transcriptional co-activators and transcription factors with acute exercise. FASEB journal : official publication of the Federation of American Societies for Experimental Biology 19 (8), 986-988 
33 Dressel, U. et al. (2003) The peroxisome proliferator-activated receptor beta/delta agonist, GW501516, regulates the expression of genes involved in lipid catabolism and energy uncoupling in skeletal muscle cells. Molecular endocrinology 17 (12), 2477-2493

Barres, R. et al. (2009) Non-CpG methylation of the PGC-1alpha promoter through DNMT3B controls mitochondrial density. Cell metabolism 10 (3), 189-198

Barres, R. et al. (2012) Acute exercise remodels promoter methylation in human skeletal muscle. Cell metabolism 15 (3), 405-411

Aoi, W. et al. (2010) The microRNA miR-696 regulates PGC-1\{alpha\} in mouse skeletal muscle in response to physical activity. American journal of physiology. Endocrinology and metabolism 298 (4), E799-806 Jager, S. et al. (2007) AMP-activated protein kinase (AMPK) action in skeletal muscle via direct phosphorylation of PGC-1alpha. Proceedings of the National Academy of Sciences of the United States of America 104 (29), 12017-12022

38 Puigserver, P. et al. (2001) Cytokine stimulation of energy expenditure through p38 MAP kinase activation of PPARgamma coactivator-1. Molecular cell 8 (5), 971-982

39 Wright, D.C. et al. (2007) Exercise-induced mitochondrial biogenesis begins before the increase in muscle PGC-1alpha expression. The Journal of biological chemistry 282 (1), $194-$ 199

40 Lerin, C. et al. (2006) GCN5 acetyltransferase complex controls glucose metabolism through transcriptional repression of PGC-1alpha. Cell metabolism 3 (6), 429-438

41 Rodgers, J.T. et al. (2005) Nutrient control of glucose homeostasis through a complex of PGC1alpha and SIRT1. Nature 434 (7029), 113-118

42 Canto, C. et al. (2010) Interdependence of AMPK and SIRT1 for metabolic adaptation to fasting and exercise in skeletal muscle. Cell metabolism 11 (3), 213-219

43 Philp, A. et al. (2011) Sirtuin 1 (SIRT1) deacetylase activity is not required for mitochondrial biogenesis or peroxisome proliferator-activated receptor-gamma coactivator-1alpha (PGC1alpha) deacetylation following endurance exercise. The Journal of biological chemistry 286 (35), 30561-30570

44 Goto, M. et al. (2000) CDNA Cloning and mRNA analysis of PGC-1 in epitrochlearis muscle in swimming-exercised rats. Biochemical and biophysical research communications 274 (2), 350354

45 Baar, K. et al. (2002) Adaptations of skeletal muscle to exercise: rapid increase in the transcriptional coactivator PGC-1. FASEB journal : official publication of the Federation of American Societies for Experimental Biology 16 (14), 1879-1886

46 Terada, S. et al. (2002) Effects of low-intensity prolonged exercise on PGC-1 mRNA expression in rat epitrochlearis muscle. Biochemical and biophysical research communications 296 (2), 350-354

47 Terada, S. and Tabata, I. (2004) Effects of acute bouts of running and swimming exercise on PGC-1alpha protein expression in rat epitrochlearis and soleus muscle. American journal of physiology. Endocrinology and metabolism 286 (2), E208-216

48 Pilegaard, H. et al. (2003) Exercise induces transient transcriptional activation of the PGC1alpha gene in human skeletal muscle. The Journal of physiology 546 (Pt 3), 851-858

49 Coffey, V.G. et al. (2006) Early signaling responses to divergent exercise stimuli in skeletal muscle from well-trained humans. FASEB journal : official publication of the Federation of American Societies for Experimental Biology 20 (1), 190-192

50 Vissing, K. et al. (2005) Are exercise-induced genes induced by exercise? FASEB journal : official publication of the Federation of American Societies for Experimental Biology 19 (1), 94-96

51 Mathai, A.S. et al. (2008) Rapid exercise-induced changes in PGC-1alpha mRNA and protein in human skeletal muscle. Journal of applied physiology 105 (4), 1098-1105 
52 Russell, A.P. et al. (2003) Endurance training in humans leads to fiber type-specific increases in levels of peroxisome proliferator-activated receptor-gamma coactivator-1 and peroxisome proliferator-activated receptor-alpha in skeletal muscle. Diabetes 52 (12), 2874-2881

Tadaishi, M. et al. (2011) Effect of exercise intensity and AICAR on isoform-specific expressions of murine skeletal muscle PGC-1alpha mRNA: a role of beta(2)-adrenergic receptor activation. American journal of physiology. Endocrinology and metabolism 300 (2), E341-349

54 Miura, S. et al. (2008) Isoform-specific increases in murine skeletal muscle peroxisome proliferator-activated receptor-gamma coactivator-1alpha (PGC-1alpha) mRNA in response to beta2-adrenergic receptor activation and exercise. Endocrinology 149 (9), 4527-4533

Zhang, Y. et al. (2009) Alternative mRNA splicing produces a novel biologically active short isoform of PGC-1alpha. The Journal of biological chemistry 284 (47), 32813-32826

Little, J.P. et al. (2010) Acute endurance exercise increases the nuclear abundance of PGC1alpha in trained human skeletal muscle. American journal of physiology. Regulatory, integrative and comparative physiology 298 (4), R912-917

57 McGee, S.L. and Hargreaves, M. (2004) Exercise and myocyte enhancer factor 2 regulation in human skeletal muscle. Diabetes 53 (5), 1208-1214

58 Safdar, A. et al. (2011) Exercise increases mitochondrial PGC-1alpha content and promotes nuclear-mitochondrial cross-talk to coordinate mitochondrial biogenesis. The Journal of biological chemistry 286 (12), 10605-10617

59 Gibala, M.J. et al. (2012) Physiological adaptations to low-volume, high-intensity interval training in health and disease. The Journal of physiology 590 (Pt 5), 1077-1084

60 Burgomaster, K.A. et al. (2008) Similar metabolic adaptations during exercise after low volume sprint interval and traditional endurance training in humans. The Journal of physiology 586 (1), 151-160

61 Terada, S. et al. (2005) Effects of high-intensity intermittent swimming on PGC-1alpha protein expression in rat skeletal muscle. Acta physiologica Scandinavica 184 (1), 59-65

62 Gibala, M.J. et al. (2009) Brief intense interval exercise activates AMPK and p38 MAPK signaling and increases the expression of PGC-1alpha in human skeletal muscle. Journal of applied physiology 106 (3), 929-934

63 Perry, C.G. et al. (2010) Repeated transient mRNA bursts precede increases in transcriptional and mitochondrial proteins during training in human skeletal muscle. The Journal of physiology 588 (Pt 23), 4795-4810

64 Little, J.P. et al. (2011) An acute bout of high-intensity interval training increases the nuclear abundance of PGC-1alpha and activates mitochondrial biogenesis in human skeletal muscle. American journal of physiology. Regulatory, integrative and comparative physiology 300 (6), R1303-1310

65 Hood, M.S. et al. (2011) Low-volume interval training improves muscle oxidative capacity in sedentary adults. Medicine and science in sports and exercise 43 (10), 1849-1856

66 Little, J.P. et al. (2010) A practical model of low-volume high-intensity interval training induces mitochondrial biogenesis in human skeletal muscle: potential mechanisms. The Journal of physiology 588 (Pt 6), 1011-1022

67 Nordsborg, N.B. et al. (2010) Relative workload determines exercise-induced increases in PGC-1alpha mRNA. Medicine and science in sports and exercise 42 (8), 1477-1484

68 Egan, B. et al. (2010) Exercise intensity-dependent regulation of peroxisome proliferatoractivated receptor coactivator-1 mRNA abundance is associated with differential activation of upstream signalling kinases in human skeletal muscle. The Journal of physiology 588 (Pt 10), $1779-1790$

69 Serpiello, F.R. et al. (2012) Repeated sprints alter signaling related to mitochondrial biogenesis in humans. Medicine and science in sports and exercise 44 (5), 827-834

70 Brocca, L. et al. (2012) The time course of the adaptations of human muscle proteome to bed rest and the underlying mechanisms. The Journal of physiology 590 (Pt 20), 5211-5230 
71 De Filippis, E. et al. (2008) Insulin-resistant muscle is exercise resistant: evidence for reduced response of nuclear-encoded mitochondrial genes to exercise. American journal of physiology. Endocrinology and metabolism 294 (3), E607-614

72 Sriwijitkamol, A. et al. (2007) Effect of acute exercise on AMPK signaling in skeletal muscle of subjects with type 2 diabetes: a time-course and dose-response study. Diabetes 56 (3), 836848

73 Ringholm, S. et al. (2011) Bed rest reduces metabolic protein content and abolishes exerciseinduced mRNA responses in human skeletal muscle. American journal of physiology. Endocrinology and metabolism 301 (4), E649-658

74 Nagatomo, F. et al. (2012) The effects of running exercise on oxidative capacity and PGC1alpha mRNA levels in the soleus muscle of rats with metabolic syndrome. The journal of physiological sciences : JPS 62 (2), 105-114

75 Li, L. et al. (2011) Mitochondrial biogenesis and peroxisome proliferator-activated receptorgamma coactivator-1alpha (PGC-1alpha) deacetylation by physical activity: intact adipocytokine signaling is required. Diabetes 60 (1), 157-167

76 Lee-Young, R.S. et al. (2011) Obesity impairs skeletal muscle AMPK signaling during exercise: role of AMPKalpha2 in the regulation of exercise capacity in vivo. International journal of obesity 35 (7), 982-989

77 Leng, Y. et al. (2004) Effects of insulin, contraction, and phorbol esters on mitogen-activated protein kinase signaling in skeletal muscle from lean and ob/ob mice. Diabetes 53 (6), 14361444

78 Koistinen, H.A. et al. (2003) Aberrant p38 mitogen-activated protein kinase signalling in skeletal muscle from Type 2 diabetic patients. Diabetologia 46 (10), 1324-1328

79 Handschin, C. (2009) The biology of PGC-1alpha and its therapeutic potential. Trends in pharmacological sciences 30 (6), 322-329

80 Summermatter, S. et al. (2010) Peroxisome proliferator-activated receptor \{gamma\} coactivator 1\{alpha\} (PGC-1\{alpha\}) promotes skeletal muscle lipid refueling in vivo by activating de novo lipogenesis and the pentose phosphate pathway. The Journal of biological chemistry 285 (43), 32793-32800

81 Choi, C.S. et al. (2008) Paradoxical effects of increased expression of PGC-1alpha on muscle mitochondrial function and insulin-stimulated muscle glucose metabolism. Proceedings of the National Academy of Sciences of the United States of America 105 (50), 19926-19931

82 Summermatter, S. et al. (2012) PGC-1alpha Improves Glucose Homeostasis in Skeletal Muscle in an Activity-Dependent Manner. Diabetes

83 Summermatter, S. and Handschin, C. (2012) PGC-1alpha and exercise in the control of body weight. International journal of obesity

84 Little, J.P. et al. (2011) Low-volume high-intensity interval training reduces hyperglycemia and increases muscle mitochondrial capacity in patients with type 2 diabetes. Journal of applied physiology 111 (6), 1554-1560

85 Handschin, C. (2010) Regulation of skeletal muscle cell plasticity by the peroxisome proliferator-activated receptor gamma coactivator 1alpha. Journal of receptor and signal transduction research 30 (6), 376-384 


\section{Figure Legends}

Figure 1. Schematic representation of the signal pathways regulating PGC-1 $\alpha$ expression and activity in skeletal muscle cells. (A) Under pathological conditions such as a sedentary life style and obesity, PGC-1 $\alpha$ expression is impaired due to aberrant activity and expression of negative and positive regulators (red circles) of this coactivator. In contrast, skeletal muscle contraction during low- and high-intensity exercise lead to the activation and inhibition of a subset of essential (blue circles) and accessory (grey circles) molecules involved in the control of the PGC-1 $\alpha$ promoter activity, in addition to its post-translational modifications and thus protein activity. Consequently, physical activity results in the activation and up-regulation of this coactivator, thereby promoting an improvement of skeletal muscle metabolic fitness. Black and red arrows show activation and inhibition, respectively. (B) PGC$1 \alpha$ drives the activation of different putative transcription factors (TF), regulating thus different aspects of muscle physiology. In this figure, some of the most relevant effects of PGC-1 $\alpha$ on skeletal muscle are shown (for more details, see ref. [85]). Abbreviations: ERR $\alpha$, estrogen-related receptor $\alpha$; NRF1/2, nuclear respiratory factor 1 and 2; FoxO1/3, forkhead box 01/3; TFAM, mitochondrial transcription factor A; MEF2, myocyte enhancer factor 2; PPAR, peroxisome proliferator-activated receptor; ROR $\alpha / \gamma$, retinoic acid receptor-related orphan receptor $\alpha / Y$; LXR $\alpha$, liver $X$ receptor $\alpha$; Nuclear factor-kappaB, NFKB; RE, TF response elements. 


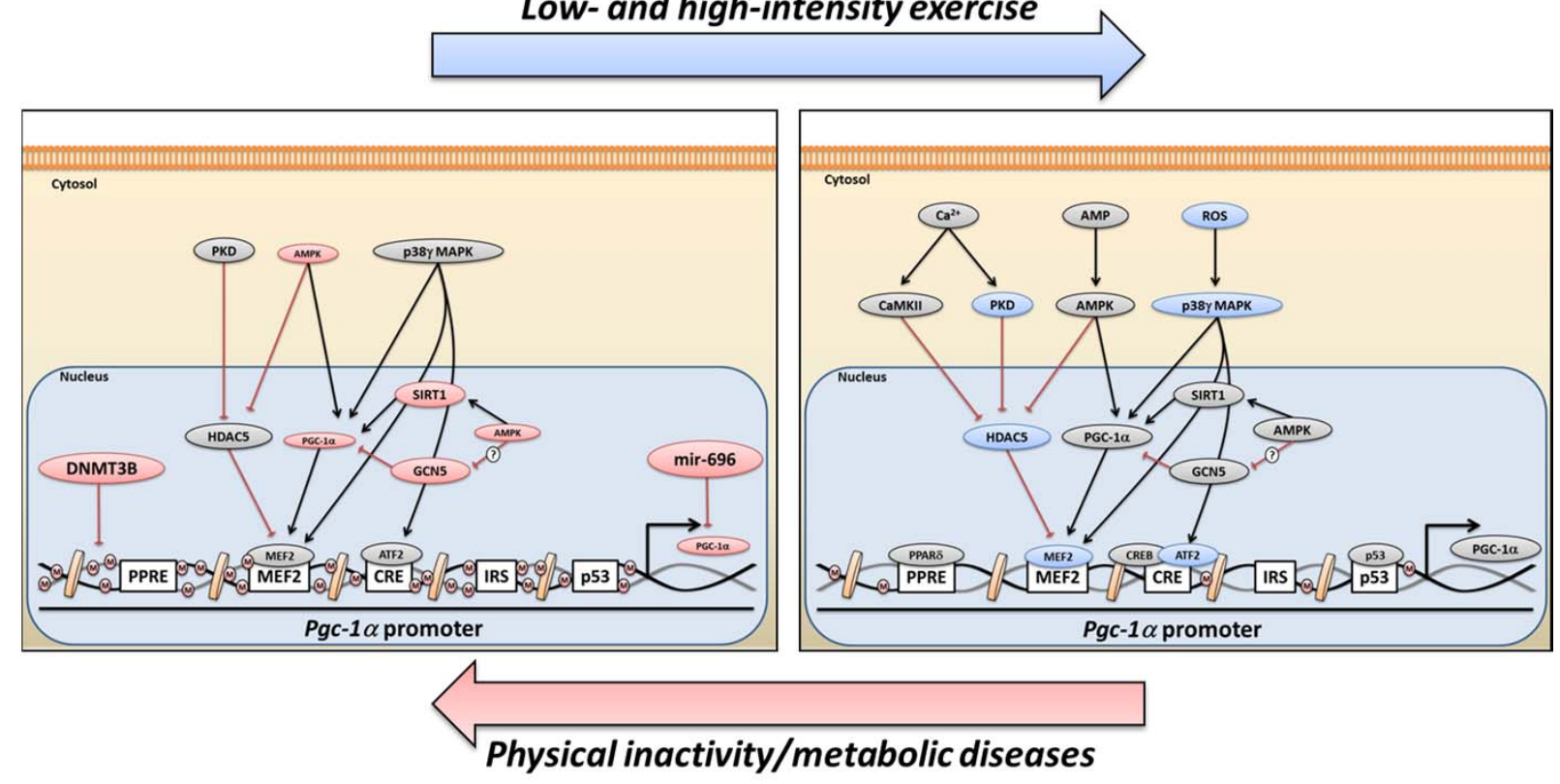

B

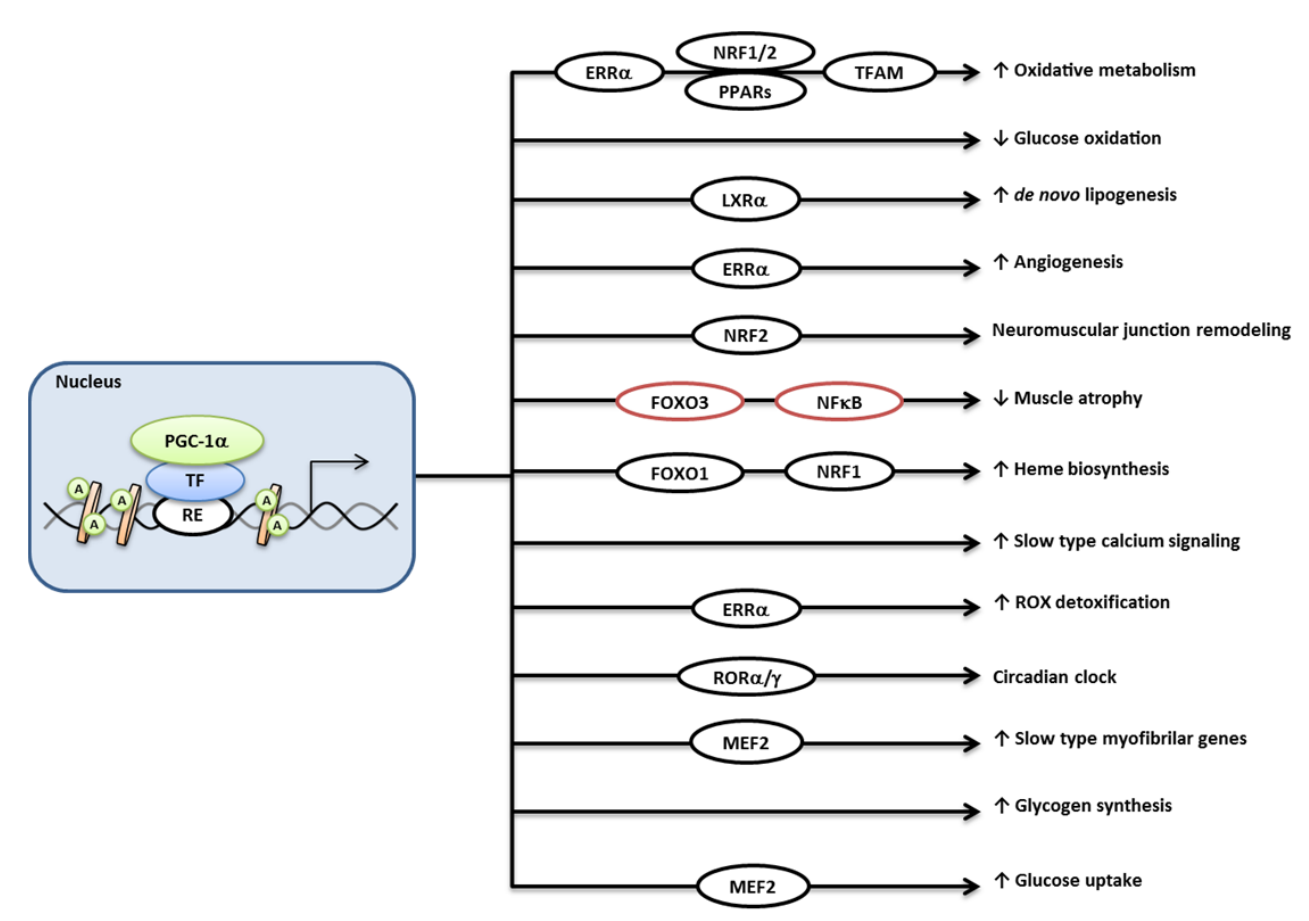

